\section{APRENDER A EMPRENDER EN LAS UNIVERSIDADES}

\author{
María Ripollés \\ Departamento de Administración de Empresas y Marketing. \\ Organización de empresas, Universitat Jaume I \\ mripolle@emp.uji.es
}

\section{LEARNING ENTREPRENEURSHIP AT UNIVERSITIES}

\begin{abstract}
This paper aims to think about the suitability of different pedagogical approaches for teaching entrepreneurship in universities. The major challenge in relation to entrepreneurship education is the need to balance the training in business knowledge needed for the exploitation of entrepreneurial opportunities with the training in the "art" of being an entrepreneur needed for the exploration of entrepreneurial opportunities. The main competences related to the "soft" side of entrepreneurial capabilities are creativity and social capabilities. Two teaching methods are presented which overcome this challenge and can facilitate teaching entrepreneurship at universities.
\end{abstract}

KEY WORDS: Entrepreneurship education; entrepreneurship at universities; entrepreneurship learning.

\section{INTRODUCCIÓN}

La creación de nuevas empresas tiene una repercusión directa en el progreso económico y social de cualquier comunidad tal y como ponen de manifiesto numerosas investigaciones cientificas como, por ejemplo, el GEM (www. gemconsortium.org). Esta realidad no ha pasado desapercibida a los responsables políticos. Distintas organizaciones internacionales, como la OCDE, o la EU (OCDE, 2008; EU Expert Group, 2008), consideran que la formación en competencias emprendedoras es importante para el progreso de las naciones; especialmente la formación universitaria. La razón estriba en que el emprendimiento universitario se asocia con la creación de nuevas empresas intensivas en conocimiento, por lo que se le considera base de la generación de empleo de calidad a largo plazo (Bird, 2002/2003).

El estudiantado también valora positivamente la formación universitaria en competencias emprendedoras ya que les proporciona el saber hacer necesario para crear su propio
RESUMEN: En este trabajo se pretende reflexionar sobre la idoneidad de distintas metodologías didácticas para la formación de emprendedores en las universidades. El principal desafío que deben superar estas técnicas está relacionado con la necesidad de compatibilizar la necesaria formación en conocimientos que requiere la explotación de oportunidades de negocio con la formación en el "arte" de ser emprendedor que requiere la exploración de oportunidades de negocio. Así además de aprender diversas técnicas estratégicas para ser emprendedor es necesario que se incentive la creatividad y el saber gestionar sus relaciones personales adecuadamente (networking capabilities). Se presentan dos técnicas didácticas que permiten superar este desafío y contribuir a la formación integral de emprendedores en las universidades.

PALABRAS CLAVE: Emprender en las universidades; capacidades emprendedoras; aprendizaje emprendedor.

puesto de trabajo y salir así de una situación de marginalidad social o laboral previa. En situaciones de crisis económica es menos arriesgado depender de uno mismo para salir de una situación de paro que depender de la voluntad y el saber hacer de otros (Jack y Anderson, 1999).

Las empresas también están interesadas en que en las universidades se fomenten competencias emprendedoras tales como la creatividad y la innovación. En opinión de Penrose (1995), la capacidad de las empresas para utilizar sus recursos de manera emprendedora les facilita la creación de mecanismos de protección frente a sus competidores $y_{1}$ por lo tanto, la creación de rentas a largo plazo. Por otro lado, centrarse en la explotación de los recursos existentes puede conducir a las empresas a una miopía directiva y al estancamiento (Kor y Mahoney, 2004).

La mayoría de responsables y docentes universitarios también comparten, aunque de manera muy tímida, la creencia de que es necesario que se cambie la "forma de hacer universidad" y se potencien las cualidades emprendedoras 
de manera trasversal en todas las titulaciones y grados que se imparten en las universidades (Zabalza, 2003-2004). El profesorado universitario tiende a primar la formación específica de cada disciplina en detrimento de la formación emprendedora, entre otros motivos por una falta de desconocimiento por su parte de cómo enseñar su disciplina de manera emprendedora. Además, en los sistemas de evaluación de la docencia del profesorado no se contempla la utilización de manera efectiva metodologías docentes que promuevan la innovación y el desarrollo de oportunidades de negocio en las materias docentes que imparten. Los sistemas de evaluación del profesorado contemplan su contribución científica y su compromiso con la generación de spin-off a partir de los resultados de sus investigaciones, pero no si el profesorado universitario es capaz de aportar una visión de sus enseñanzas aplicada al aprovechamiento de oportunidades de mercado y despertar el espíritu emprendedor del estudiantado universitario.

La postura de los responsables de las universidades es algo más favorable hacia la formación emprendedora en casi todas las universidades españolas. La necesidad de contribuir a la creación de empresas es considerada parte de la tercera misión de las universidades. Sin embargo, la materialización de esta misión con políticas y programas de actuación concretos no está tan extendida y depende de la existencia de cátedras institucionales o de patrocinio que suelen desarrollar esta formación mediante títulos propios de formación continua y/o de post-grado.

Una revisión de las características de estos programas de formación permite detectar algunas carencias. Estas carencias limitan los resultados de dichos programas y cuestionan su capacidad como motor de creación de spinoff universitarias. En este sentido, cabe señalar el hecho de que la formación para emprender ha sido abordada casi exclusivamente por profesores estrechamente relacionados con las áreas de dirección de empresas, quienes han extrapolado los métodos didácticos utilizados para trasmitir conocimientos en gestión de empresas a la formación de emprendedores, asimilando dos realidades tan distantes como la administración de empresas consolidadas y el desarrollo de una idea de negocio hasta convertirla en una realidad empresarial. La utilización de la clase magistral, de lecturas especializadas, de casos prácticos o las charlas con empresarios ha predominado en las aulas universitarias; sin embargo, y puesto que emprender es también, y sobre todo, un proceso creativo, existen dudas de la utilidad de estos métodos didácticos convencionales (Garavan y O'Cinneide, 1994). Algunos autores van más allá y señalan que la utilización de estos métodos puede incluso llegar a inhibir el espíritu emprendedor del estudiantado (Bager, 2011), sobre todo porque otorgan al estudiante un papel pasivo en el proceso de aprendizaje.

La necesidad de formar a emprendedores universitarios y la aparente falta de efectividad de las metodologías docentes tradicionales justifica las llamadas que desde distintos ámbitos se está haciendo a favor de un cambio en la forma de abordar la docencia universitaria en competencias universitarias y creación de empresas desde las universidades. Cambio que implica que el estudiantado universitario adopte un papel activo y participe en el proceso de aprendizaje emprendedor.

En este artículo se pretende reflexionar sobre la idoneidad de las diferentes metodologías didácticas a la hora de formar personas emprendedoras en las universidades. Para ello, analizaremos la idoneidad de las metodologías didácticas tradicionales para fomentar estas capacidades. Describiremos los desafíos que plantea la formación de emprendedores en las universidades. Y, por último, presentaremos una propuesta integradora de diferentes métodos didácticos que pueden contribuir a paliar el déficit de las metodologías didácticas tradicionales.

\section{LOS DESAFíOS DE ENSEÑAR A EMPRENDER EN LAS UNIVERSIDADES}

Basándonos en la conceptualización de emprender de Shane y Venkataraman (2000) en este artículo asumimos que para emprender es necesario que exista una persona creativa (o un equipo de personas) -en adelante persona emprendedora- capaz de identificar una nueva oportunidad económica y de conseguir los recursos necesarios para explotarla. Además, como cualquier directivo, la persona emprendedora tiene que ser capaz de gestionar adecuadamente los recursos de que dispone para que su nueva empresa desarrolle una ventaja competitiva a largo plazo. Recordemos que este saber hacer no identifica a la persona emprendedora sino al directivo y se enseña en casi todas las universidades españolas. 
Los métodos didácticos utilizados tradicionalmente en dirección de empresas no son del todo apropiados para formar a personas emprendedoras porque se basan, precisamente, en la racionalidad, la previsión y el orden en la trasmisión de conocimientos perfectamente codificables. El objetivo de los diferentes programas formativos en dirección de empresas es el adiestramiento en la utilización de determinados procesos y/o técnicas funcionales creando especialistas funcionales capaces de gestionar adecuadamente cualquier departamento de una empresa. Recursos didácticos como la lección magistral, el método del caso o las charlas con empresarios suelen ser los más utilizados en estos programas. La eficacia de estos métodos es elevada si la trasmisión de conocimientos es el objetivo de estos cursos; sin embargo cuando la trasmisión de conocimientos es también el medio entonces no son del todo apropiados (Garavan y O'Cinneide, 1999). De hecho, se ha afirmado que los métodos mencionados limitan la creatividad y favorecen una representación errónea el mundo real (Fiet, 2000).

El emprendedurismo requiere encontrar y definir problemas y carencias que existen en una sociedad que deben resolverse de manera creativa en la actividad de creación de una empresa. Para ello es necesario la utilización de métodos didácticos que involucren directamente al estudiantado en el proceso de trasmisión de conocimientos emprendedores (Bager, 2011). Métodos que despierten el lado "creativo" de la persona emprendedora y que le permitan gestionar adecuadamente sus relaciones sociales.

Los métodos didácticos tradicionales, propios de los programas de gestión o dirección de empresas, han recibido críticas, pues, por no ser suficientes para enseñar a emprender en las universidades y relegar al estudiante a un papel secundario en el proceso de aprendizaje. Según Fiet (2000) los principales inconvenientes de dichos métodos para el fomento de las competencias emprendedoras son que:

- Se da por hecho que el estudiantado se encontrará con las mismas circunstancias que se ejemplifican en los casos o en las lecturas, con lo que se están generalizando comportamientos que no tienen por qué cumplirse en otros contextos.

- Se está asumiendo que hay un comportamiento emprendedor ideal, el ejemplificado en los casos o lectu- ras. La realidad nos dice que el mejor comportamiento emprendedor dependerá de las circunstancias concretas de cada caso.

- Se está aceptando la hipótesis de que la información del pasado permite entender el futuro. Esta hipótesis no se sostiene en entornos turbulentos como los actuales.

- Se está asumiendo que el conocimiento emprendedor es una información pública por lo que todos los individuos podrían convertirse en emprendedores de éxito ya que poseen esa información.

Estos inconvenientes parecen aconsejar la utilización de métodos didácticos participativos en la formación de personas emprendedoras (Bird, 2002/2003). Estos métodos se basan en la premisa de que para aprender es necesario que el estudiantado experimente nuevos roles de forma que se puedan cambiar comportamientos. El aprendizaje participativo implica que el alumnado gestione su propio proceso de aprendizaje. Que tenga autonomía para definir los objetivos de aprendizaje, contenidos, método, evaluación y la definición de los resultados de aprendizaje (Can$d y, 1991)$. En este sentido el papel del profesorado dista mucho del rol tradicional de trasmisor de conocimientos. El profesorado se convierte en un gestor del proceso de aprendizaje del estudiantado. Pasando de ser el instructor a ser el guía y el asesor. Su papel debe ser el de proporcionar esquemas conceptuales e información; debe también motivarles para pensar de forma creativa y transgresora; debe animarles a buscar nuevas fuentes de información y asesorarles ofreciéndoles nuevas alternativas, nuevas vías de desarrollo de forma que el estudiantado vaya adquiriendo nuevo conocimiento a través de la experimentación. La necesidad de este cambio de metodología docente en la formación de personas emprendedoras está en línea con las nuevas exigencias docentes de la era post-Bolonia (López, 2005).

Sin embargo y aunque es necesario la utilización de nuevas técnicas docentes que potencien la parte irracional de la creación de empresas, para una persona emprendedora un balance y/o una investigación de mercados tiene el mismo significado que para un directivo de una gran empresa por eso deben combinarse con los métodos tradicionales. Son necesarios también métodos que permitan la trasmisión de conocimiento, de forma que el estudiantado adquiera los instrumentos estratégicos necesarios para definir un plan de negocio. 
El dilema, por lo tanto, estriba en cómo compaginar la necesaria formación en conocimientos que requiere la explotación de oportunidades de negocio con la formación en el "arte" de ser emprendedor que implica la identificación de nuevas oportunidades (Jack y Anderson, 1999) y la obtención de los recursos necesarios para explotarlos. Son necesarios métodos que permitan la efectiva integración entre la trasmisión de conocimientos propia de una docencia tradicional y la participación activa del estudiantado en su aprendizaje.

En el siguiente apartado expondremos dos métodos didácticos que cumplen con estos requisitos. Estos métodos son complementarios y pueden utilizarse para desarrollar los conocimientos necesarios para analizar la viabilidad de una idea de negocio, elaborar un plan de acción y para ejercitar las capacidades emprendedoras que hemos señalado en el apartado anterior. Estos métodos son: el contrato de aprendizaje y el proyecto tutorizado.

\section{Aprender a Emprender: el contrato DE APRENDIZAJE Y EL PROYECTO TUTORIZADO}

\section{Aprendizaje voluntario a través del contrato de aprendizaje}

Aunque todavía no hay datos de la utilización de este método en las universidades para formar a personas emprendedoras, Bird (2002/2003) destaca que este método implica centrarse en el estudiantado a la hora de diseñar y ejecutar un proyecto de aprendizaje, al mismo tiempo que requiere del profesorado un rol de facilitador del desarrollo del proyecto.

La autora propone un metodología que se basan en la premisa de que es la persona que está aprendiendo competencias quien debe adquirir nuevo concomimiento, adoptar un nuevo rol y cambiar su autoconcepto y comportamiento; por lo que el aprendizaje debe estar autodirigido. El papel del instructor se centra en proporcionar marcos conceptuales, guia, información, opinión y motivación para desarrollar el nuevo conocimiento y comportamiento. Las etapas para aplicarlo a la hora de enseñar las competencias emprendedoras se resumen en (Bird, 2002/2003):
1. El profesorado debe explicar con detalle las distintas competencias asociadas a las personas emprendedoras. Se acompañan de ejemplos de empresarios famosos, se utilizan videos y demás métodos didácticos tradicionales, etc. El objetivo es que el estudiantado puedan ver en acción esas competencias.

2. El estudiantado debe concretar las competencias que quieren desarrollar. Esta definición se hace de manera conjunta con el profesorado, de forma que éste se asegure de que se van a trabajar determinadas competencias.

3. El estudiantado ha de autoevaluarse en relación con las competencias seleccionadas.

4. El estudiantado debe seleccionar aquellas áreas de las competencias seleccionadas en las que necesitan mejorar y elaboran un plan de trabajo para poder desarrollarlas. Este plan de trabajo debe ser concreto y realista, y debe estar consensuado con el profesorado.

5. El estudiantado y el profesorado deben establecer la forma en la que se evaluarán dichas competencias.

\section{Aprendizaje por proyecto tutorizado: el plan de negocio como herramienta de aprendizaje}

De manera complementaria al contrato de aprendizaje los formadores de emprendedores cuentan con el plan de negocio como herramienta docente siempre y cuando supere las limitaciones relacionadas con la necesidad analítica $a$ priori de los participantes y no tenga como único objetivo la redacción del documento. Para ello se debe animar al estudiantado a participar en un proceso de aprendizaje cuyo camino es tan importante como su finalidad: un plan de negocio que sea realista y susceptible de convertirse en una nueva empresa. De tal forma que el proceso de aprendizaje permite al estudiantado considerar diversas actitudes, valores, conocimientos, roles y motivaciones de cara a iniciar una actividad empresarial. Prestando la atención adecuada al proceso el estudiantado puede identificar las competencias necesarias para su proyecto empresarial. El proceso de elaboración del plan de negocio conlleva el reto de desarrollar dichas competencias y otras que hubieran quedado fuera de un programa estándar de aprendizaje.

La experiencia desarrollada en la Universitat Jaume I en la formación de personas emprendedoras a través de cursos 
de postgrado ha demostrado que esta metodología permite equilibrar el aprendizaje autodirigido y el experimental. En dichos cursos el proceso se ha desarrollado mediante las siguientes etapas:

1. A cada estudiante se le asigna un tutor que deberá guiarle y asesorarle en su proceso de aprendizaje. Puede ser el mismo tutor con el que el estudiante ha firmado el contrato de aprendizaje de determinadas capacidades emprendedoras.

2. En las clases presenciales todo el alumnado trabaja las diferentes herramientas estratégicas que le permita definir una idea innovadora y desarrollarla hasta convertirla en una empresa. Se trata de clases orientadas a la acción. Se explica cómo utilizar las herramientas estratégicas que permiten el análisis de la viabilidad de una idea de negocio y a continuación el estudiantado debe aplicarlas a sus ideas de negocio.

3. El estudiantado, con su tutor, definirá cuáles son las áreas del plan de negocio en las que existen carencias y se definirá un itinerario de clases tutorizadas con distintos especialistas, de forma que el estudiantado pueda corregir con éxito las deficiencias y conocer perspectivas diferentes del proceso de creación de empresas a las del profesorado.

4. El estudiantado elaborará un plan de negocio bajo la supervisión de su tutor. El tutor es el responsable de orientar al estudiantado a la hora de buscar las fuentes de información que necesita para elaborar su plan de negocio.

5. El estudiantado deberá presentar su plan de negocio ante determinados foros económicos y empresariales. Estas presentaciones tienen un doble objetivo: por un lado permiten al estudiantado obtener información muy valiosa que puede contribuir a una mejora del plan de negocio; y por otro lado le permite ejercitar las habilidades de comunicación y sociales.

\section{A MODO DE CONCLUSIÓN}

Al principio de este trabajo nos planteábamos reflexionar sobre la idoneidad de las distintas metodologías didácticas a la hora de formar a emprendedores universitarios. La conclusión a la que hemos llegado es que estas metodologías deben facilitar la integración entre la necesidad de trasmitir conocimientos y la de desarrollar las distintas competencias emprendedoras. Hemos presentado dos metodologías que de manera complementaria permiten resolver este dilema. Ahora bien, no existen resultados estadísticos que permitan evaluar la efectividad de su aplicación, por lo que se trata de propuestas de trabajo cuyos resultados han sido estimados únicamente de forma intuitiva. Se hace pues necesario el diseño de investigaciones que permitan medir la eficacia de diversas metodologías docentes para el fomento del emprendedurismo universitario. Los problemas que deben resolverse a la hora de diseñar estas investigaciones son de diversa índole y no tienen una fácil solución. Se requiere que se especifiquen claramente los objetivos que se persiguen con los distintos programas de formación. Objetivos que son diferentes atendiendo al tipo de audiencia considerado: estudiantado, profesorado, responsables administrativos, colectivo empresarial. A la hora de establecer criterios para evaluar la efectividad en el cumplimiento de estos objetivos se debe tener en cuenta que la incidencia de los programas de formación de personas emprendedoras es también a largo plazo y no es fácil establecer criterios que permitan medir la efectividad de estos programas a lo largo del tiempo, ni obtener la información requerida.

\section{BIBLIOGRAFÍA}

Bager, T. (2011): "Entrepreneurship Education and new venture creation: a comprehensive approach", K. Hindle y K. Klyver (Eds.), Handbook of research on new venture creation, Edgar Elgar, Cheltenham, UK., pp. 299-315.
Bird, B. J. (2002/2003): "Learning entrepreneurship competences: The self-direct learning approach", International Journal of Entrepreneurship Education, 2 (1), pp. 41-59.

Candy, P. C. (1991). Self-direction for lifelong learning: A comprehensive guide to theory and practice, San Francisco: Jossey-Bass. 
EU (Comisión Europea): Dirección electrónica: http://ec.europa.eu

Fiet, J. (2000): "The theoretical side of teaching entrepreneurship", Journal of Business Venturing, 16, 1-24.

Garavan, T. N. y O'Cinneide, B. (1994): "Entrepreneurship education and training programmes: A review and evaluation - Part 2", Journal of European Industrial Training, 18 (11), pp. 13-22.

GEM (Global Entrepreneurship Monitor) (2010): Informe GEM España 2010. Obtenido en: http://www.ie.edu/gem/ img/informes/61.pdf.

Jack, S. L. y Anderson, A. R. (1999): "Entrepreneurship education within the enterprise culture. Producing reflective practitioners", International Journal of Entrepreneurial Behaviour \&t Research, 5 (3), pp. 110-125.

Kor, Y. Y. y Mahoney, J. T. (2004). Edith Penrose's (1959): "Contributions to the resource-based view of the firm", Journal of Management Studies, 41 (1): 183-91.

López, F. (2005): Metodología participativa en la enseñanza Universitaria, Narcea.

Observatorio UJ (Universitat Jaume I de Castellón): http://www.uji.es/CA/serveis/ocie/acil/prog/obser/esp/
OCDE (Organisation for Economic Co-operation and Development): Dirección electrónica: http://www.oecd.org.

Penrose E. (1995): "Research on the business firm limits to the growth and size of firms", The American Economic Review, 45 (2), pp. 531-543.

Shane, S. y Venkatraman, S. (2000): "The promise of entrepreneurship as a field of research", Academy of Management Review, 25 (1), pp. 217-26.

Zabalza, M. (2003-2004): Innovación en la enseñanza universitaria, Contextos Educativos, pp. 113-136. 